\title{
Euroanalysis XVI-challenges in modern analytical chemistry
}

\author{
Slavica Ražić
}

Published online: 8 March 2012

(C) Springer-Verlag 2012

In the International Year of Chemistry, Belgrade was the meeting place for almost 600 participants of Euroanalysis XVI, from 57 countries from all over Europe and overseas. As a broad-spectrum conference on analytical chemistry, held biennially with its venue rotating between European countries, Euroanalysis has established itself as a regular forum of the national societies represented in the Division of Analytical Chemistry of the European Association for Chemical and Molecular Sciences (EuCheMS). The 16th European Conference on Analytical Chemistry, held in September 2011, was hosted by the Division of Analytical Chemistry of the Serbian Chemical Society (SCS), and fully supported by the SCS and its president Ivanka Popović. The Congress Centre SAVA in Belgrade, located in one of the modern quarters of New Belgrade but still very close to the old historic and charming city centre, provided an excellent venue for the conference, enabling networking at its best on both the scientific and social level.

An attractive scientific program was presented under the motto "Challenges in Modern Analytical Chemistry" and there was much to be learnt about the trends and perspectives in the different areas of the analytical sciences. Ten plenary lectures built the framework of the conference. How far can one go with all the available analytical tools? "Diving Deep into the Chemistry of the Human Brain", the lecture of Jonas Berquist from Uppsala University (Sweden), was a good example of the use of novel techniques for analysis of complex samples, the isolation and enrichment of the non-soluble portion of the proteome - membrane-bound proteins from brain tissue. The techniques applied (CPE, 1D gel electrophoresis, in-gel digestion with RP-nanoLC, ESI and high-resolution MS-MS, or direct digestion with RP-nanoLC, MALDITOF/TOF-MS or high-resolution IEF with ESI-nanoLCMS-MS) are very promising in the study of tissues from the human central nervous system or animal models of neurological diseases. His presentation was awarded the Robert Kellner Lecture prize, established by the Division of EuCheMS in memory of the efforts and contributions of the late Robert Kellner, from Vienna University of Technology, and traditionally sponsored by Springer, Heidelberg.

In his lecture "The Role of Accurate Mass Measurement in Chemical, Analytical and Medical Mass Spectrometry" G. Brenton outlined how high-resolution mass spectrometry

Published in the special issue Euroanalysis XVI (The European Conference on Analytical Chemistry) with guest editor Slavica Ražić.

S. Ražić $(\bowtie)$

Department of Analytical Chemistry, Faculty of Pharmacy,

University of Belgrade,

Vojvode Stepe 450,

11221 Belgrade, Serbia

e-mail: slavica.razic@pharmacy.bg.ac.rs 
has become a central technique for analytical measurements, particularly as a superior detector in specific areas such as proteomics and pharmaceuticals. On the other hand, combined with appropriate separation techniques, ICP-MS provides not only reliable elemental information but also reveals speciation of small and large molecules (e.g., proteins in biological systems) and for nanoparticles and nanostructures also. Some attractive applications of ICP-MS and GD-MS (TOF) were presented by another award recipient, Alfredo Sanz-Medel (University of Oveido, Spain). His lecture "The Expanding Scope of Analytical Atomic Spectrometry: Isotopes, Elements, Molecules and Nanoparticles via Mass Spectrometry" was designated the EuCheMS Lecture 2011 for his outstanding achievements. In the lecture "The Power of Selectivity in Chromatography" W. Lindner showed how powerful chromatography could be. Selectivity adjustment drives the success of liquid chromatography and enables even very complex enantioselective separations and monoclonal antibody purification. "New Tools for the Analytical Laboratory" were proposed by G. Hieftje, among which two sources were prominent: flowing atmospheric pressure afterglow (FAPA), useful for ambient mass spectrometry, and solution-cathode glow discharge (SCGD), applied to the determination of trace-metal concentrations in solutions. A forthcoming challenge is the development of analytical methods enabling determination of the interactions of metals with the biomolecules which constitute the genome, proteome, metabolome, and other "omes" in cell, tissue, or organism. Combined techniques based on the coupling of high-resolution separation, for example HPLC, capillary electrophoresis, or gel electrophoresis, with elemental (ICP-MS) and molecular (ES-MS-MS) mass spectrometry offer the possibility of high-throughput acquisition of metallomics information for many biological systems ("Biological Trace Element Analysis, Speciation and Metallomics", by R. Lobinski). Food authentication is another challenging application area, which was demonstrated for food analysis of various metalobomic-based samples ("Metabolomic Fingerprinting/Profiling Employing High Resolution Mass Spectrometry: a Challenging Strategy in Food Analysis", by J. Hajslova).

An exciting lecture on the integration of membrane and proteins into electronic devices to provide biosensors attaining detection limits in the low-ppt range was delivered by L. Torsi ("Bio-electronic Devices as Ultra-sensitive Analytical Sensors"). Continuing with this topic, it was shown that aptamers could be regarded as a valid alternative to antibodies or other bio-mimetic receptors for the development of biosensors for applications based on molecular recognition, as analytical, diagnostic, and therapeutic tools ("Peptide and Oligonucleotide
Aptamers as New Ligands for Analytical Chemistry", by M. Mascini). Novel optical sensing strategies, establishing new-generation optical sensing platforms in biodiagnostics were presented by B. Mizaikoff ("Miniaturized mid-infrared sensors - How small is still useful?")

Bioanalytical topics attracted many researchers from analytical laboratories and outside the community of analytical chemists. Many issues of global importance were recognized, for example requirements for quantification of genetically modified constituents in food and fodder samples, fast and reliable screening tests in medicinal diagnostics, new methods and methodologies for drugs, hormones, and vitamins analysis, and identification and quantification of hazardous substances in biotic and abiotic environmental compartments. Although improved hyphenated techniques (HPLC-DAD-MS, HPLCUV-MS-MS, ...) dominate in food analysis, HPLC-fluorescence and UHPLC-PDA were confirmed as accurate and reliable enough for specific target analytes in complex food samples. Chemometrics is almost unavoidable in data treatment of complex data sets and also offers vast possibilities in signal treatment for improving the detection power of numerous analytical methods. Forensics and archeometry were also challenging fields both on the border between natural sciences and humanities. In general, both methods and problem-oriented contributions created an excellent atmosphere for exchange of knowledge in analytical sciences. All that is reflected in the papers presented in this special issue.

An adequate education in analytical sciences opens vast possibilities, so the session dedicated to Education in analytical chemistry at the beginning of the 21 st century raised many questions important in higher education, building the career and lifelong learning programs for expanding knowledge, developing and improving skills, and increasing the abilities of analytical chemists. All in all, 135 oral and 560 poster presentations, featuring core topic areas of analytical chemistry and, especially, the impressive number of young scientists (more than 200) created an excellent atmosphere for enthusiastic discussions on cutting-edge analytical chemistry. It is important to acknowledge that the quality of posters was generally high and an international jury had a difficult task selecting the best six, the authors of which each received a Springer poster prize.

In parallel with the scientific program, the participants widely used the opportunity to attend several lunch symposia and participate in vivid discussions in the exhibition hall where companies and scientific organizations presented their novel concepts of smarter technology and the latest innovations and solutions. Networking and creating new contacts and co-operation are what traditionally make Euroanalysis attractive. 
Additionally, the Euroanalysis party, in a restaurant on a small river island, and several excursions organized during and after the conference, contributed to a lasting overall impression of the Euroanalysis days in 2011.

We wish to thank the sponsors for their support, colleagues and students for their assistance and enthusiasm to make Euroanalysis 2011 a success. However, all the organizational tasks and demands would not have been accomplished without professional support by Congrexpo, and its representative Olivera Blagojević, who has shown, in the last few years and during the conference, that "problems" were not problems, but could be solved with kind words and friendly smile.

Concerning this special issue, I am very grateful to the reviewers for their thorough and on-time reviews of the manuscripts and to the editorial team of Analytical and Bioanalytical Chemistry for their great cooperation.

The preparations for Euroanalysis 2013 in Warsaw (Poland) by Maciej Jarosz and Ewa Bulska have already commenced, in order to continue this successful series of conferences.

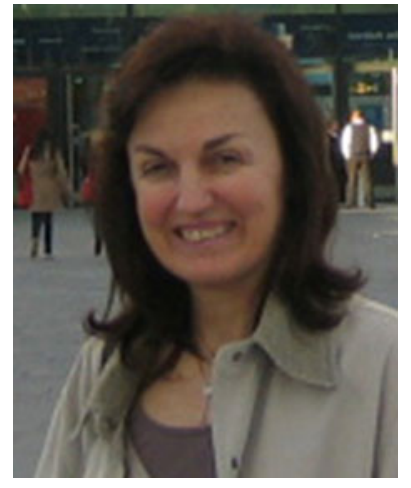

Slavica Ražić is a professor in the Department of Analytical Chemistry of the Faculty of Pharmacy of the University of Belgrade. She served as the Head of the Institute of Analytical Chemistry for two terms in the last decade. Her main research interests are analysis of environmental and biological samples, method development, and monitoring trace and minor elements by use of atomic absorption and emission spectrometry ( $\mathrm{F}-\mathrm{AAS}$, F-AES, GF-AAS, ICP-AES, and ICP-MS) combined with contemporary sample-preparation techniques and chemometric methods of analysis. Elucidation of the bioavailability, mobility, distribution, and accumulation of elemental species in different environmental compartments, and their affect on human health, is a constant analytical challenge. Her current research interests were recently extended to GC-MS analysis of active organic compounds in plants important in phytopharmacy. Since 2002, she has chaired the Division of the Analytical Chemistry of the Serbian Chemical Society (SCS) and also served as its representative in the Division of Analytical Chemistry of the European Association of Chemical and Molecular Sciences (EuCheMS). Slavica Ražić is a member of the editorial board of the Journal of the Serbian Chemical Society and is a subeditor of Analytical Chemistry. 